\title{
The Rise of Complexity in Nature
}

\author{
Eric J. Chaisson \\ Wright Center for Science Education \\ Tufts University, Medford, MA 02155 USA, \\ USA Email: eric.chaisson@tufts.edu \\ URL: www.tufts.edu/as/wright_center
}

\begin{abstract}
Cosmic evolution-change, broadly construed-has become a powerful unifying factor in all of science, underlying the rise of complexity throughout Nature writ large. That complexity can be defined and quantified for a whole hierarchy of ordered structures by examining the rate at which normalized energy flows through open, non-equilibrium, thermodynamic systems, from quark to quasar, microbe to mind.
\end{abstract}

\section{Introduction}

Science currently has three great frontiers: the very big, the very small, and the very complex. Throughout a quarter-century of research and teaching, I have contributed in small ways to each of these perplexing areas. More fundamentally, I have tried to cross their boundaries and explore commonalities among themin particular, to unite astrophysics and biochemistry in a grand interdiscipline called "cosmic evolution". Such work helps to provide an intellectual blueprint for the emerging subject of astrobiology or bioastronomy.

Some colleagues explore superstrings, eleven dimensions, and entirely new physics in their quest for unification. By contrast, I embrace the concept of evolution in the four-dimensional world that clearly surrounds us, without necessarily introducing any new science. But my vision of evolution is broad and inclusive, incorporating Darwinism within a much wider scheme designed to understand change on all spatial and temporal scales.

Cosmic evolution is the study of the many varied developmental and generative changes among radiation, matter, and life throughout the history of the Universe. As such, it does not pertain merely to astronomical systems, but to all organized structures, including life. Hence, physical, biological, and cultural evolution is each a part of this integrated, evolutionary Weltanschauung. In short, mine is an attempt to include life naturally, perhaps inevitably, within the larger cosmological perspective.

\section{New Results}

Three years ago, at the Bioastronomy '99 meeting, I quantitatively sketched a way to embed living systems within standard, big-bang cosmology (Chaisson 
2000; see also Chaisson 1998 and 1999). I have since bolstered and extended that analysis in a book-length monograph (Chaisson 2001).

The crux of my argument is that all ordered structures can be empirically judged by examining the flow of energy through open, non-equilibrium systems. By normalizing to the mass of each system the energy fluxing through those systems, the complexity of ordered structures-from galaxies to stars to planets to life-can be compared "on the same page". That complexity is related to normalized energy flow should not surprise us since, in an expanding, unequilibrated Universe, it is free energy that thermodynamically drives order from chaos, in fact increasingly so with time post decoupling at $\sim 10^{6}$ years.

All such complex systems can be shown to be in accord with the second law of thermodynamics; entropy decreases in such systems locally, yet increases by larger amounts in their surrounding environments globally. However, entropy measures are tricky to specify numerically, as are the information contents of such systems. Hence another reason to adopt an energetics agenda, given that energy-acquired, stored, or expressed-is well-defined, physically intuitive, and measurable. Energy-the ability to do work, or to cause change-is the most universal currency known in natural science.

Here, I extend my earlier analysis, summarizing more empirical findings of normalized energy flows through a wide spectrum of physical, biological, and cultural systems. With few exceptions, free energy rate density proves a useful quantitative indicator of complexity, indeed of the rise of complexity from big bang to humankind.

Figure 1 plots the rise of free energy rate density for various ordered systems, connecting horizontal histograms covering the time durations when those systems prevailed in Nature. Much as expected, that rise in complexity has been rapid in the last few billion years, and especially so since the Cambrian epoch. The solid curve approximates the increase in normalized energy flow that best characterizes order, form, and structure throughout the history of the Universe. The circled inserts show greater detail of further calculations of the free energy rate density passing through a variety of stars, planets, plants, animals, society, and machines.

Consider stars, for example. Stars most assuredly grow in complexity as their thermal and elemental gradients steepen with time; more information is needed to describe them as they age. And so does their energy rate densities increase temporally, from protostars at "birth", to main-sequence stars at mature middle "life", to red giants near "death". This is simply the change in light/mass ratio used by astronomers. On and on, the cycle churns; build up, break down, change-a process we call stellar "evolution" minus any genes, inheritance, or overt function, for these are the value-added qualities of biological evolution that go well beyond evolution of astronomical systems.

Consider plants and animals. Here, with some exceptions, rising complexity is evident throughout evolution, again exemplified by energy-flow diagnostics. This is the change in specific metabolic rate used by biologists. Life forms require the acquisition of more energy per unit mass than does any star, and increasingly so with biological evolution. Onward across the bush of life-cells, tissues, organs, organisms-we find much the same story. Starting with life's precursor molecules and all the way up to plants, animals, and brains, the same general trend typifies 


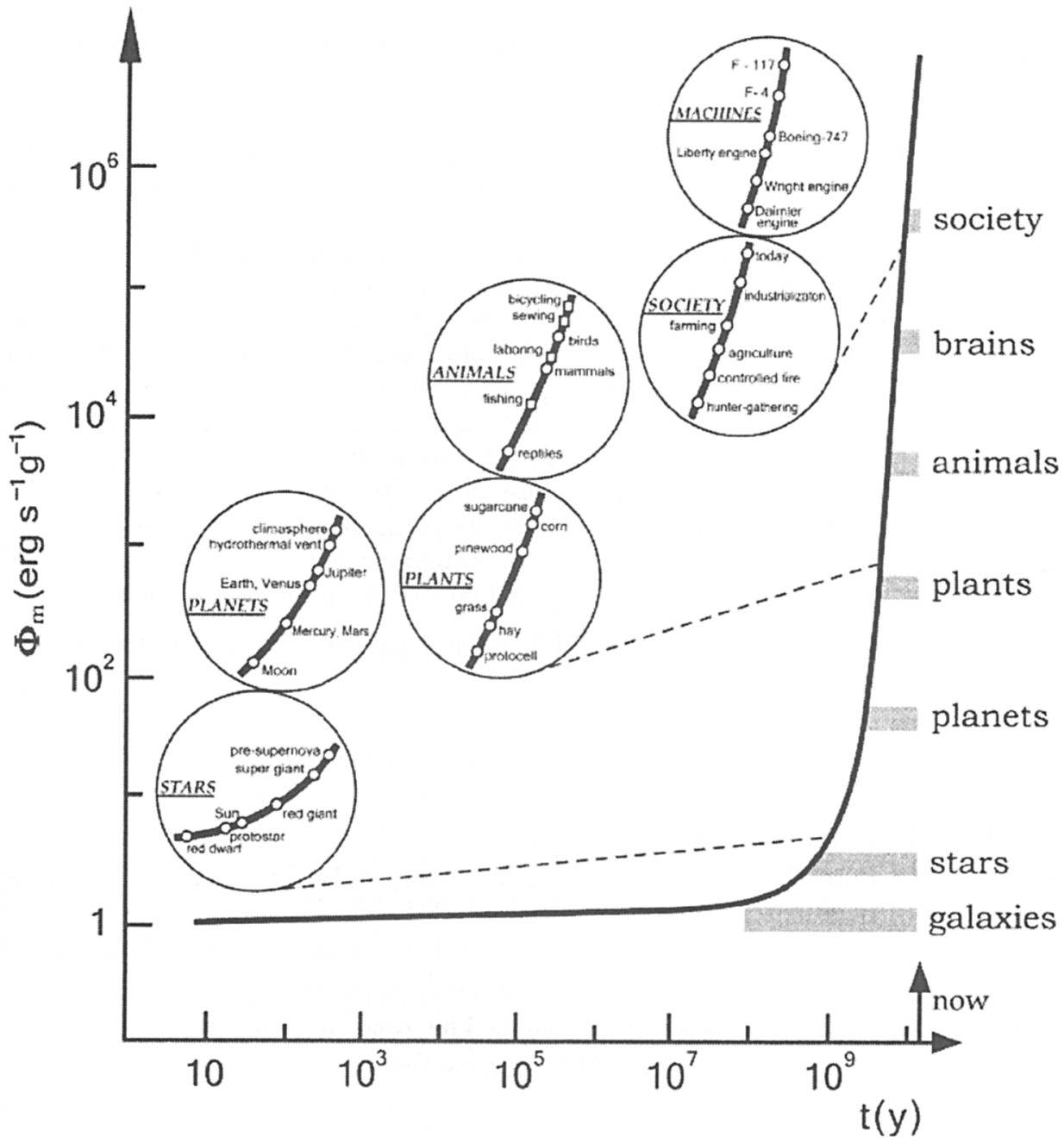

Figure 1. Free energy rate density, $\Phi_{m}$, calculated here in units of $\mathrm{erg} \mathrm{s}^{-1} \mathrm{~g}^{-1}$ and plotted on a log-log scale against time, has risen dramatically for a wide spectrum of ordered systems that have emerged over the course of the past fourteen billion years. 
animate life forms as for inanimate stars and planets: The greater the perceived complexity of the system, the greater the flow of energy density through that system-either to build it, or to maintain it, or both.

Finally, consider society and its cultural evolution. Once again, we can trace social advancement, mainly energy consumption, for a variety of human-related achievements among our hominid ancestors. Quantitatively, that same energy rate density increases from hunter-gatherers of a million years ago to agriculturists of the past several thousand years, to industrialists of contemporary times. And here, along the road to civilization, as well as among the bricks, machines, and chips we've built, energy is the driver. Energy rate density clearly rises with the increasing complexity of today's gadget-rich society-as any engineer, who worries about power density, will attest.

More than any other single factor, energy flow would seem to be a principal means whereby Nature's diverse systems have naturally, perhaps inevitably, generated complexity in an expanding Universe. Some of those systems have harnessed optimum energies and thus evolved impressive complexity typical of life, mind, and consciousness-including, not least, a sentient curiosity sufficient to enable us to look back and probe past events that transpired along the earlier arrow of time. In turn, energy, specifically humankind's use of it wisely and optimally, will likely guide our future fate along the continuing arrow of time.

An extensive educational product on the subject of cosmic evolution, suitable for introductory college and advanced secondary-school courses, is available on the Wright Center site: www.tufts.edu/as/wright_center/cosmic_evolution.

Acknowledgments. This work has been supported in part by the Fondation H. Dudley Wright of Geneva, Switzerland.

\section{References}

Chaisson, E. J. 1998, BioSystems, 46, 13

Chaisson, E. J. 1999, in Quest for a Unified Theory of Information ed. Hofkirchner, (Amsterdam: Gordon \& Breach), 231

Chaisson, E. J. 2000, in ASP Conf. Ser. 213, Bioastronomy '99: A New Era in the Search for Life ed. G. Lemarchand \& K. Meech, 35

Chaisson, E. J. 2001, Cosmic Evolution: The Rise of Complexity in Nature, (London: Harvard University Press Cambridge) 